\title{
Eukaryotes under a new light
}

Proteorhodopsins are light-driven proton pumps that harvest energy from light to help bacteria and archaea grow and survive when nutrients are scarce. Now, Slamovits and colleagues show that bacteriaderived proteorhodopsins are present in some marine protists.

During a genomic analysis of various marine dinoflagellates, the authors sequenced $>18,000$ expressed sequence tags (ESTs) from Oxyrrhis marina, a predatory microorganism that is abundant in shallow waters, and found several sequences encoding proteins that were highly similar to bacterial proteorhodopsins. Interestingly, similar ESTs were also obtained from two other dinoflagellates, Pyrocystis lunula and Alexandrium tamarense. The conservation of these proteorhodopsin genes among distantly related dinoflagellates suggests that the genes may have been horizontally transferred from marine bacteria around the time of origin of the dinoflagellates. Moreover, the O. marina ESTs included transcripts encoding a protein that is highly similar to bacterial carotenoid dioxygenases and therefore may have a role in the synthesis of the carotenoid retinal, a prosthetic group found in proteorhodopsins. The dinoflagellate may bacteria-derived proteorhodopsins are present in some marine protists.

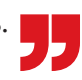
have acquired this enzyme either horizontally from bacteria or vertically from an ancestral plastid.

The proteorhodopsin sequences were the most highly represented nuclear genes in the EST survey of

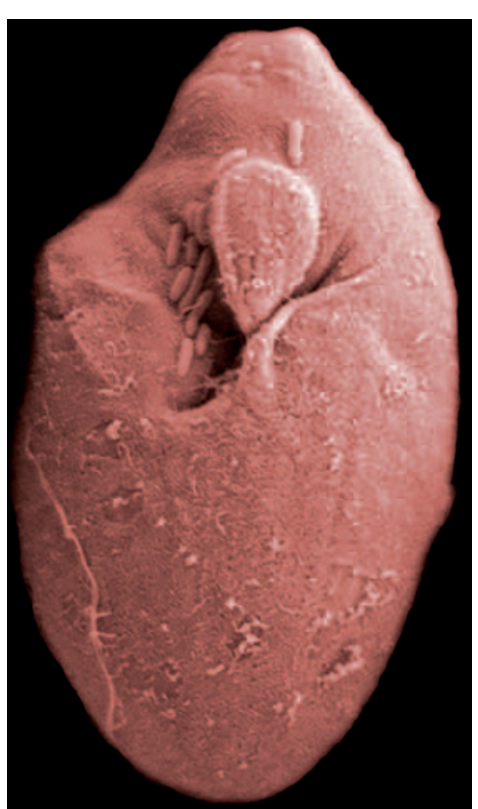

Colourized scanning electron micrograph of the dinoflagellate Oxyrrhis marina. Image courtesy of S. Breglia, University of British Columbia, Vancouver, Canada.

O. marina, which may explain the bright pink colour of the microorganism, as proteorhodopsins typically exhibit a red colour. The $O$. marina proteorhodopsins probably possess seven transmembrane domains as well as the key residues that are characteristic of the proteorhodopsin family, as indicated by alignment with bacterial proteorhodopsins and secondary-structure prediction. Furthermore, the presence of a particular residue (Leu109) suggests that the O. marina proteins are tuned to maximally absorb green light, which is consistent with the ecology of this organism.
In bacteria, proteorhodopsins generate a proton gradient across the plasma membrane, but eukaryotic cells possess complex membranous systems and, therefore, there are many compartments in which a gradient can be established. To determine the subcellular location of the O. marina proteorhodopsins, an antibody was developed against their deduced carboxyl termini. Using immunofluorescence microscopy, the authors showed that the proteins were distributed unevenly within the cytosol, in an unidentified fraction of the endomembrane system.

These results indicate that the proteins identified in O. marina are proteorhodopsins of bacterial origin, but their function remains unknown. The authors hypothesize that the proteins may generate a proton gradient in endomembrane compartments to force the reverse functioning of vacuolar ATPases or pyrophosphatases (which usually use ATP or pyrophosphate to produce a proton gradient), leading to lightdependent synthesis of energy-rich ATP or pyrophosphate. Alternatively, the proteorhodopsins could replace or supplement the normal function of these vacuolar enzymes by using light to acidify digestive vacuoles.

Cesar Sanchez

ORIGINAL RESEARCH PAPER Slamovits, C. H. et al. A bacterial proteorhodopsin proton pump in marine eukaryotes. Nature Commun. 2, 183 (2011) FURTHER READING Fuhrman. J. A., Schwalbach, M. S. \& Stingl, U. Proteorhodopsins: an array of physiological roles? Nature Rev. Microbiol. 6, 488-494 (2008) 